\title{
Intelligent Manager of Scientific Knowledge Based in Gamification (Gicci-Game)
}

\author{
Anthony Martínez, Mag. ${ }^{1}$, Juan Valera Mariscal, Mag. ${ }^{2}$, Yenny Ochomogo, Lic. ${ }^{1}$, Ramfis Miguelena, Dr. ${ }^{1}$ \\ ${ }^{1}$ Universidad Tecnológica de Panamá, Panamá, anthony.martinez@utp.ac.pa, yenny.lopez@utp.ac.pa, \\ ramfis.miguelena@utp.ac.pa \\ ${ }^{2}$ Gamification Inside, juan@valera-mariscal.com
}

\begin{abstract}
Today we see how prevalent the need for updated information representing the experience and skills of an organization, educational institution, company or group of experts on a topic with the aim of which are documented both explicit knowledge (which is simple record and report) and tacit knowledge (which can be understood only by one person and therefore is not easy to record or document). Here's where we consider appropriate to set up a smart manager to apply scientific knowledge gamification techniques in order to manage information and provide a mechanism to create, store and access it.
\end{abstract}

Keywords- Gamification, knowledge base, research, engagement, platform, web design.

Digital Object Identifier (DOI): http://dx.doi.org/10.18687/LACCEI2015.1.1.171

ISBN: 13 978-0-9822896-8-6

ISSN: $2414-6668$

$1^{\text {th }}$ LACCEI Annual International Conference: “Engineering Education Facing the Grand Challenges, What Are We Doing?” July 29-31, 2015, Santo Domingo, Dominican Republic ISBN: 13 978-0-9822896-8-6

ISSN: $2414-6668$

DOI: http://dx.doi.org/10.18687/LACCEI2015.1.1.171 


\title{
Intelligent manager of scientific knowledge based in Gamification (Gicci-Game)
}

\author{
Anthony Martínez, Magister ${ }^{1}$, Juan Valera Mariscal, Magister ${ }^{2}$, Yenny Ochomogo, Licenciada ${ }^{1}$, \\ Ramfis Miguelena, Doctor ${ }^{1}$ \\ ${ }^{1}$ Universidad Tecnológica de Panamá, Panamá, anthony.martinez@utp.ac.pa, yenny.lopez@utp.ac.pa, \\ ramfis.miguelena@utp.ac.pa \\ ${ }^{2}$ Gamification Inside, juan@valera-mariscal.com
}

\begin{abstract}
Today we see how prevalent the need for updated information representing the experience and skills of an organization, educational institution, company or group of experts on a topic with the aim of which are documented both explicit knowledge (which is simple record and report) and tacit knowledge (which can be understood only by one person and therefore is not easy to record or document). Here's where we consider appropriate to set up a smart manager to apply scientific knowledge gamification techniques in order to manage information and provide a mechanism to create, store and access it.
\end{abstract}

Keywords-Gamification, knowledge base, research, engagement, platform, web design.

\section{INTRODUCCIÓN}

En la actualidad, se puede observar como el uso de la tecnología se ha incrementado de forma considerable en diversos ámbitos, tales como la investigación, la educación superior y a nivel profesional. Es así como estos escenarios han generado nuevos espacios de interacción, modelos de representación de información y gestión de contenidos en general, haciendo posible la comunicación entre saberes, cultura y sociedad; propiciando así la denominada revolución científica-investigativa.

Como investigadores en el área de las tecnologías de la información y las comunicaciones consideramos oportuno la creación de un repositorio o biblioteca de conocimiento científico que permita al usuario ser receptor y/o divulgador de información en diversas áreas del conocimiento que le interese y así inspirarse para sus propios proyectos de innovación o facilitar el camino a otros; así como también pueda inducir a los integrantes de su grupo a las diversas actividades que se generen a través de la demanda de ayuda, la creación de casos, y la resolución de problemas o retos científicos que puedan ser valorados y apoyados por otros investigadores; aplicando para ello técnicas de gamificación que permitan la creación de un escenario donde los usuarios puedan interactuar.

Es así como podemos citar la definición de José Luis Ramírez en su libro Gamificación: Mecánicas de juegos en tu vida personal y profesional [1], en donde nos dice que la gamificación es aplicar estrategias (pensamientos y mecánicas) de juegos en contextos no jugables, ajenos a los juegos, con el fin de que las personas adopten ciertos comportamientos. Es decir, se aplican elementos y reglas de juego con el objetivo de captar, atrapar usuarios, trabajadores y clientes con la finalidad de generar compromiso (engagement), fomentar conductas deseadas y resolver problemas.

\section{CONCEPTO SOBRE Gestión DE CONOCIMIENTO}

Antes de continuar adelante, es necesario conocer en que consiste la gestión de conocimiento. En una organización cuando se habla de gestión del conocimiento, se refieren a un proceso o una situación que permita trasferir los conocimientos o experiencias de una persona o grupo de personas a otras personas pertenecientes a una misma organización u empresa.

Y es ahí donde radica el principal problema, ya que nos referimos a personas y de cómo van trasmitir o compartir algo que no es fácil de trasmitir ni saber a quién trasmitir: la información y habilidades [2].

Por ello, podemos indicar que la gestión del conocimiento no es más que el proceso por el cual una organización, facilita la transmisión de informaciones y habilidades a sus empleados, de forma ordenada, efectiva y eficiente, tal como podemos observar en la Fig. 1.

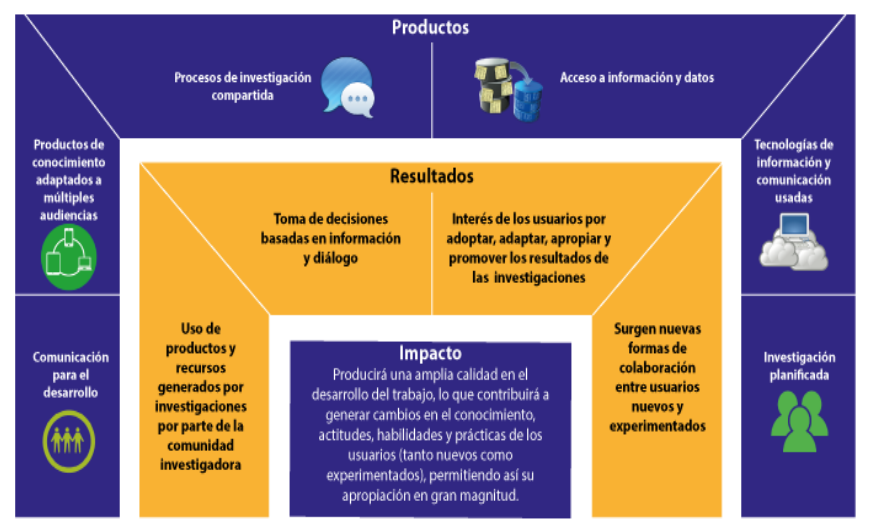

\section{GESTION DEL CONOCIMIENTO}

Fig. 1 Diagrama de Gestión del Conocimiento

En tal sentido, en los componentes de la gestión del conocimiento que son la información y las habilidades podemos observar que la primera puede ser recolectada, procesada y almacenada por los sistemas de información de la organización; mientras que las segundas son un tanto más complicadas de transmitir por el simple hecho de que implican la adquisición de un nuevo conocimiento para el que las recibe.

$13^{\text {th }}$ LACCEI Annual International Conference: "Engineering Education Facing the Grand Challenges, What Are We Doing?” July 29-31, 2015, Santo Domingo, Dominican Republic DOI: http://dx.doi.org/10.18687/LACCEI2015.1.1.171 


\section{ESTADO DEL ARTE DE LA GESTIÓN DEL CONOCIMIENTO}

Basados en los límites geográficos que circunscriben a este proyecto, los cuales son Panamá y España, se han obtenido pocos resultados en relación a la existencia de iniciativas basadas en modelos de gestión de conocimiento en donde se apliquen técnicas innovadoras para la consecución de los objetivos planteados basados en la recolección e intercambio de información entre sus miembros y que los mismos logren alcanzar objetivos y cumplir metas para lograr un estatus dentro de la comunidad a la que pertenecen. He aquí donde nace la iniciativa de plantear un modelo de gestión de conocimiento científico basado en las técnicas de gamificación a nivel web, que permita centralizar, gestionar y desarrollar el conocimiento existente en la sociedad, a través de sus miembros y sus actividades, generando mayor interés y engagement (enganche), de forma que se convierta en una herramienta útil, sea utilizada por sus miembros y ello conduzca inexorablemente a un desarrollo personal y profesional de los mismos y, por consiguiente, a una mejora en el conocimiento científico y capital intelectual.

En base a lo antes expuesto, se observa que el uso de la tecnología se ha incrementado de forma considerable en diversos ámbitos, tales como la investigación, la educación superior y a nivel profesional. Es así como estos escenarios han generado nuevos espacios de interacción, modelos de representación de información y gestión de contenidos en general, haciendo posible la comunicación entre saberes, cultura y sociedad; propiciando así la denominada revolución científica-investigativa. En tal sentido, como investigadores en el área de las tecnologías de la información y las comunicaciones consideramos oportuno la creación de un repositorio o biblioteca de conocimiento que permita al usuario ser receptor y/o divulgador de información en diversas áreas del conocimiento que le interese y así inspirarse para sus propios proyectos de innovación o facilitar el camino a otros; así como también pueda inducir a los integrantes de su grupo a las diversas actividades que se generen a través de la demanda de ayuda, la creación de casos, y la resolución de problemas o retos científicos que puedan ser valorados y apoyados por otros investigadores.

En este sentido, sabemos que, el conocimiento es un motor esencial en el incremento de la productividad de un país y en la mejora del bienestar social de sus ciudadanos. Es por ello que, en la sociedad basada en el conocimiento, la transferencia del mismo desde el ámbito científico y académico a la sociedad en general y a las organizaciones en particular, va adquiriendo cada vez mayor importancia.

Uno de los mecanismos fundamentales para la mejora de la capacidad tecnológica e investigadora en la sociedad es a través de la transferencia de ciencia y tecnología desde las entidades públicas y privadas de investigación, los cuales son organismos que constituyen la infraestructura de soporte a la innovación de cualquier sistema nacional y/o regional de innovación. La capacidad innovadora de un país o región no depende únicamente de su esfuerzo cuantitativo en I+D y de su infraestructura tecnológica (centros e instituciones que realizan actividades innovadoras), sino de la interacción entre las empresas, las administraciones públicas y otros agentes, como por ejemplo universidades y otros centros públicos y privados de investigación. En tal sentido se puede indicar que las empresas, aunque requieran los conocimientos desarrollados por las instituciones científicas, constituyen los agentes esenciales de la innovación.

Por otro lado, a nivel mundial encontramos ejemplos relacionados con la gestión del conocimiento tales como Foldit [3], Aprendizaje colaborativo, Livemocha, Liveops, entre otros.

Fold.it se trata de un proyecto de la Universidad de Washington para usar la inteligencia colectiva. Para ello diseñaron una plataforma de modelado 3D a modo de videojuego para atraer a una comunidad cuyo objetivo es diseñar proteínas, o más concretamente, la idea es analizar y proponer formas de plegamiento de las proteínas, cuestión que resulta clave a la hora de luchar contra ciertas enfermedades. De este modo se convertía la difícil y aburrida tarea de analizar el plegamiento óptimo de las proteínas en un divertido juego multijugador online llamado Foldit [4] [5].

A comienzos de 2012 ya había unos 240.000 inscritos, con más de 2.000 activos por semana. Se recogieron más de 70.000 propuestas para la primera parte y unas 110.000 para la segunda. Luego, los investigadores que estaban detrás del proyecto eligieron las respuestas que les parecían más prometedoras y consiguieron enzimas 18 veces más eficaces que las naturales en un tiempo record. La idea es ayudar en la investigación de enfermedades como el SIDA, Cáncer o Alzheimer. Como ejemplo, con la colaboración de miles de usuarios jugando, consiguieron en 10 días sintetizar una proteína para combatir el virus del VIH que los expertos llevaban buscando más de una década.

Un ejemplo evidente del potencial del crowdsourcing[6] y la innovación abierta para resolver problemas, en este caso de índole científica, aprovechando la inteligencia colectiva a través de la red y las ventajas de la gamificación y los juegos.

Otro ejemplo claro del uso de la gamificación en el área de ventas y aprendizaje en una empresa es My Work Community de Liveops [7].

MyWorkCommunity [8] es una plataforma gamificada para potenciar el aprendizaje y el rendimiento de los operadores de Liveops, la mayor plataforma de Call Center de Estados Unidos en la nube. La empresa con más de 20.000 operadores independientes que trabajan desde casa, con gran dispersión geográfica y dan servicio a importantes compañías de todo el mundo.

Mediante este sistema, el proceso de aprendizaje inicial de los operadores se redujo de 160 horas a 14 horas entre los participantes en relación con los que no participaron en el proyecto gamificado. Además los participantes superaron a los demás en un $23 \%$ en la gestión del tiempo de llamada y mejoraron un $9 \%$ la satisfacción del cliente. 


\section{OBJETIVOS A CUMPLIR}

Basados en lo antes expuesto, con este proyecto se busca crear un gestor inteligente de conocimiento científico aplicando técnicas de gamificación para estimular la participación, colaboración y la realización de proyectos de investigación; en donde los usuarios podrán tener acceso a lecturas, presentaciones y trabajos realizados por otros investigadores o documentos compartidos de interés clasificados por temáticas en un repositorio multimedia (texto, videos recomendados, presentaciones). De igual forma, los usuarios podrán actualizar la plataforma con el aporte de ideas, soluciones, documentos científicos, entre otros.

En primera instancia se desarrollaría una plataforma web interactiva aplicando el concepto de gamificación que permita a través de la experiencia de juegos, promover el uso de un gestor inteligente de conocimiento científico para estimular la participación, colaboración y la realización de proyectos de investigación, tanto en Panamá como en España.

Esta plataforma permitirá, entre otras cosas:

1) Establecer las tareas de interacción, así como la arquitectura de información requerida para una adecuada experiencia de usuario.

2) Establecer los elementos de juego, junto con las técnicas de la gamificación más apropiadas para la plataforma web interactiva; con el fin de motivar a los miembros del gestor de conocimiento científico para su retroalimentación.

3) Definir la arquitectura más adecuada, tanto de software como de hardware, para el despliegue de la experiencia interactiva.

4) Implementar la plataforma web interactiva junto con la generación de contenido multimedia requerido, a través de la construcción de un prototipo funcional.

5) Realizar pruebas funcionales y de usabilidad con el fin de garantizar la mayor autonomía para los miembros y futuros usuarios del gestor de conocimiento científico gamificado.

\section{BENEFICIOS Y PRINCIPALES BENEFICIARIOS}

Dentro de los principales beneficios que generará este proyecto podemos mencionar que se dotará a Panamá y España de una herramienta que atraiga la participación internacional y que dado su perfil innovador posicione a ambos países como referentes y dinamizadores de la actividad investigadora a nivel internacional. De igual forma, podemos indicar que luego de que se realice un análisis de la tipología del usuario / jugador combinado con la selección de las mecánicas y componentes gamificados adecuadamente, nos conducirá a generar acciones de alto impacto que produzcan los siguientes beneficios:

- Ayudará a generar cambios de comportamiento aplicados a diversos ámbitos dentro del gestor de conocimiento científico.

- Se obtendrán grandes cantidades de datos que posteriormente podrían ser procesados con técnicas de BigData y/o visualización.
- Facilitará el proceso de re-construcción, sistematización y apropiación del conocimiento a través de la innovación y generación de estímulos y aportes orientadores para la apropiada creación o re-creación del conocimiento adquirido.

- Este gestor de conocimientos permitirá, a través de la interacción permanente en la plataforma web interactiva, estimular la puesta en escena del conocimiento tácito (experiencia y propuesta de nuevas fórmulas para responder a limitantes del desarrollo) y contribuye con su conocimiento explícito a la construcción de un consenso cognitivo útil.

Este proyecto tendrá como principales beneficiarios a la comunidad científica-investigadora de ambos países, los docentes investigadores de instituciones académicas y la empresa privada. Es importante señalar que dentro de los beneficiarios directos de este proyecto estarán los estudiantes, ya que serán los principales receptores de esta información, la cual les servirá como material de apoyo al desarrollo de sus actividades académicas.

\section{JUSTIFICACIÓN}

A nivel mundial se ha podido observar de forma constante, como las sociedades han intentado proveer a la población de una serie de elementos y factores que en su conjunto busquen elevar y garantizar el nivel de vida de sus habitantes, a través de esquemas o modelos de desarrollo que coadyuven en una mayor y mejor calidad de vida de las sociedades que están expuestas a los grandes cambios producto de la globalización.

En tal sentido, hay que buscar afanosamente la vía más idónea para adaptarlas a las continuas transformaciones. Una de estas vías es la gestión del conocimiento científico, donde se abordan competencias relacionadas con las actitudes que facilitan crear y compartir el conocimiento, así como las que preparan para el aprendizaje a lo largo de la vida.

Para ello, se considera oportuno emplear mecánicas de juego en entornos y aplicaciones no lúdicas con el fin de potenciar la motivación, la concentración, el esfuerzo, la fidelización y otros valores positivos comunes a todos los juegos para influir y motivar a grupos de personas [9] a través de una plataforma de gestión del conocimiento científico.

\section{METODOLOGÍA}

La aplicación del enfoque lúdico en la elaboración de propuestas para la creación de espacios de aprendizaje se lleva haciendo desde hace tiempo como una forma de refuerzo para la apropiación de contenidos concretos de aprendizaje. Sin embargo, la gamificación supone una nueva perspectiva. En el entorno educativo, la gamificación puede suponer a priori el diseño de tareas y actividades usando los principios que se aplican a los juegos. Se trata de aprovechar la predisposición natural hacia el juego para mejorar la motivación hacia el aprendizaje, la adquisición de conocimientos, la transmisión de valores, el desarrollo de competencias, entre otros. Es aquí donde el uso y aplicación de herramientas tecnológicas y/o 
multimedia en este tipo de espacios, nos lleva como investigadores y docentes, a experimentar y explotar las posibilidades que ofrecen las nuevas tecnologías para su aprovechamiento didáctico en estos espacios de aprendizaje.

Por ello, consideramos que la creación de la plataforma web denominada Gestor Inteligente de Conocimiento Científico basado en Técnicas de Gamificación (GICCIGAME), debe ser realizada sobre pilares fundamentales, a saber:

- Simplicidad y Accesibilidad: Contará con un diseño fácil e intuitivo basado en el concepto one-click de modo que el usuario acceda a la información que necesita con los mínimos clics de ratón posibles.

- Experiencia de Usuario: Se tendrán en cuenta aspectos de usabilidad. El diseño será enfocado en el usuario (HCD Human Centered Design / DCP - Diseño Centrado en las Personas [10]) de modo que desde su perspectiva suponga una mejora de la experiencia de interacción y uso de la plataforma.

- Flexibilidad: Esta herramienta se adaptará totalmente a los perfiles y necesidades del usuario y de la empresa en tiempo real.

- Aprendizaje y desarrollo Iterativo: El desarrollo inicial será la plataforma funcional base a partir de la cual la información del comportamiento de los usuarios en la misma dará lugar a mejoras continuas en un proceso de iterativo ágil.

- Modularidad y escalabilidad: Se hará el desarrollo de la Plataforma por módulos. Esto permitirá la implantación por módulos, haciéndola totalmente escalable $\mathrm{y}$ permitiendo comenzar por una funcionalidad básica, para luego ir escalando hacia una plataforma con una gran cantidad de funcionalidades y apps.

- Modelo Sistémico: La herramienta será planteada partiendo de un concepto de sistema en el que cada participación de un elemento determina y afecta el comportamiento y los resultados del conjunto. Este modelo sistémico favorece el funcionamiento autónomo del sistema. Se facilita que el usuario sea autónomo y le permite la auto-gestión, auto-desarrollo, auto-motivación, auto-evaluación y auto-expansión. Al conectar los rendimientos personales con los de la comunidad mediante logaritmos de interacción social, se producirá la vinculación entre las acciones y los resultados de los usuarios para que fomente la participación y la regulación interna de la participación auto-inter usuario.

- Seguridad: Se busca que la plataforma cumpla con todos los estándares y medidas de seguridad de la información en todos los niveles.

En virtud de lo antes expuesto, consideramos que tener un modelo de Gestión del Conocimiento Científico apoyado en un entorno gamificado, sería la solución para que el conocimiento este siempre al alcance de todos, pues ofrece un espacio de integración, comunicación, colaboración y generación de soluciones con herramientas que capitalizan el conocimiento producido por los miembros en las diferentes áreas (Ver Fig. 2), permitiendo hacer más efectivas tanto las actividades como los procedimientos, porque los mismos podrán operar de manera acertada, rápida y sencilla al tener a su disposición materiales e información del entorno, el cual quedará centralizado, incrementando de esta manera el conocimiento y productividad individual mediante la utilización eficiente de los recursos.

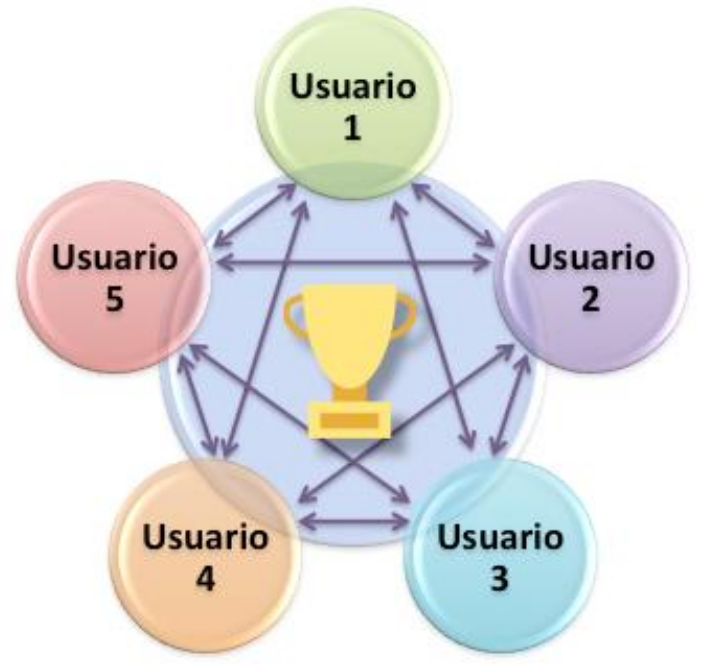

Fig. 2 El diagrama refleja que el conocimiento se intercambia en un modelo en red, donde los usuarios tienen varios roles pero que se resumen en el concepto Prosumer (prosumidor) ya que son tanto productores como consumidores de conocimiento. En este intercambio de información, y de interacciones. La gamificación actúa como un motivador de acción y generador de vínculo entre el usuario y el sistema. (El trofeo en el diagrama representa eso).

\section{RESULTADOS ESPERADOS}

El principal resultado que se espera obtener de este proyecto está el desarrollo de una plataforma tecnológica vía web que utilizará técnicas de gamificación que motivará a la participación e incrementará los comportamientos deseables dentro del sistema, como lo son la publicación de documentos, su valoración, establecimiento de lazos de colaboración, intercambio de conocimiento, entre otras cosas, dependiendo de los roles que sean asignados. Esto generará dentro de los participantes prestigio social, información y colaboración de otros expertos.

De igual forma la plataforma gamificada podrá incluir incentivos tipo: reconocimiento institucional de participación, publicaciones o selecciones más valoradas al año, retos y desafíos científicos, creativos y de conocimiento, entre otros.

Otro aspecto fundamental que aporta el diseño gamificado es la obtención y registro inmediato de métricas de actividad que permiten la verificación de los logros así como la iteración, evolución y mejora continua de la plataforma, del diseño y del propio proyecto, siendo a su vez, fuente de datos para futuras investigaciones. Se propone pues, no solamente como un proyecto final sino como un modelo pionero en el mundo y un motor impulsor de la actividad científica y de la cultura de la innovación. 
En resumen proponemos un gestor inteligente de conocimiento científico gamificado (GICCI-GAME) que estimule la colaboración, la creatividad y el intercambio entre la comunidad investigadora.

El proyecto será implementado a nivel web para que pueda ser accedido por toda la comunidad investigadora, sin importar los límites geográficos.

En consecuencia consideramos que el impacto de este proyecto recaerá en las facilidades que brindará el contar con una herramienta tecnológica a nivel web que permita agrupar por temáticas documentos, presentaciones, archivos multimedia, entre otros, para que estén a disposición de todo aquel que así lo requiera. De igual forma, esta plataforma servirá de punto de encuentro para el establecimiento de enlaces con investigadores en cualquier parte del mundo con el objetivo de realizar proyectos de investigación en conjunto para beneficio de ambas partes. Esta herramienta también permitirá crear comunidades de usuarios comprometidos en torno a los modelos de comunicación surgidos del nuevo escenario de la cibercultura y las TICs.

\section{REFERENCIAS}

[1] J. Ramírez. "Gamificación: Mecánicas de Juegos en tu vida personal y profesional”, SCLIBRO, pp. 27-28, 2014.

[2] Qué es gestión del conocimiento, Papeles de Inteligencia. http://papelesdeinteligencia.com/que-es-gestion-delconocimiento/.

[3] Blog de David Sánchez Bote. http://dsanchezbote.com/?p=1743.

[4] El país. http://sociedad.elpais.com/sociedad/2012/01/23/actualida d/1327319734_495819.html.

[5] Las proteínas. http://proteinas.org.es/juego-proteinas-foldit.

[6] ¿Qué es el Crowdsourcing y qué ventajas tiene?http://www.empresasenred.es/empresasenred/blog/ $\% \mathrm{C} 2 \% \mathrm{BFqu} \% \mathrm{C} 3 \% \mathrm{~A} 9-\mathrm{es}-\mathrm{crowdsourcing}$-y-ventajas-tiene

[7] Liveops.com. http://www.liveops.com/connect/pressroom/liveops-winsgold-for-social-learning-from-chief-learning-officermagazine.

[8] Bunchball. http://www.bunchball.com/customers/liveops.

[9] Qué es la gamificación. http://www.gamificacion.com/que-es-la-gamificacion.

[10] Diseño Centrado en la Persona. IDEO toolkit. http://www.ideo.com/work/human-centered-designtoolkit/. 